\title{
Association of Circulating Irisin Levels with Adiposity and Glucose Metabolic Profiles in a Middle-Aged Chinese Population: A Cross-Sectional Study
}

This article was published in the following Dove Press journal: Diabetes, Metabolic Syndrome and Obesity: Targets and Therapy

\author{
Ruibin Zhang ${ }^{1} * *$ \\ Tingting $\mathrm{Fu}^{2, *}$ \\ Xin Zhao ${ }^{3}$ \\ Yao Qiu ${ }^{4}$ \\ Xiaolin $\mathrm{Hu}^{5}$ \\ Hongyan $\mathrm{Shi}^{4}$ \\ Xiao Yin (iD $)^{5}$
}

'Department of Medicine, Shandong University Affiliated Jinan Central Hospital, Jinan, Shandong, People's Republic of China; ${ }^{2}$ Department of Clinical Medicine, Qilu College of Medicine, Shandong University, Jinan, Shandong, People's Republic of China; ${ }^{3}$ Department of Clinical Laboratory, Shandong University Affiliated Jinan Central Hospital, Jinan, Shandong, People's Republic of China; ${ }^{4}$ Department of Surgery, Shandong University Affiliated Jinan Central Hospital, Jinan, Shandong,

People's Republic of China; ${ }^{5}$ Department of Endocrinology and Metabolism, Shandong University Affiliated Jinan Central Hospital, Jinan, Shandong, People's Republic of China

*These authors contributed equally to this work

\begin{abstract}
Objective: The myokine irisin has been proposed to affect obesity and metabolism disorders. However, data about the association of irisin with obesity and glucose metabolic status in humans remains controversial, and limited data are available concerning the Chinese population. This study aimed to evaluate the association between serum irisin concentrations and obesity, as well as glucose metabolic and cardiovascular factors in the middle-aged physical activity-matched Chinese Han race population.
\end{abstract}

Methods: A total of 740 participants were included in this cross-sectional study, who were divided into a normal weight (NW) group, overweight/obese (OB) group, normal weight type 2 diabetes (DM-NW) group and overweight/obese diabetes (DM-OB) group, and physical activity was evaluated and matched for the four groups. Circulating irisin levels were analyzed and compared among the groups with different adiposity and glucose status. Linear regression analysis was performed to test the relationship between serum irisin and adiposity indices, glucose metabolic and other cardiovascular risk factors.

Results: Circulating irisin levels were significantly correlated with higher levels of direct and indirect adiposity markers, including BMI, waist circumference and fat mass, and other cardiovascular risk factors, such as plasma triglyceride and low-density lipoprotein cholesterol level $(\mathrm{p}<$ 0.01). Diabetes patients had lower irisin levels compared to non-diabetes participants, in either normal weight and obese group, resulting in a correlation of irisin with HbAlc, HOMA-IR and HOMA-IS $(\mathrm{p}<0.01)$. Linear regression analysis demonstrated that irisin serum concentrations were independently associated with sex, BMI, HbAlc, HOMA-IR and HOMA-IS $\left(\mathrm{R}^{2}=0.465\right)$. Conclusion: These data indicated that circulating irisin was affected by adiposity and glucose metabolism condition in the middle-aged Chinese population. The increase of irisin under conditions of obesity may indicate its physiological function to improve glucose tolerance which is often impaired in obese subjects, but this compensatory secretion of irisin seems likely to be progressing to a secretion failure once diabetes developed.

Keywords: irisin, obesity, type 2 diabetes, Chinese

\section{Introduction}

Irisin is a myokine derived from the transmembrane protein fibronectin type III domain-containing protein 5 (FNDC5), the expression of which is elevated in muscle in response to exercise in mice and humans. Irisin binds to yet unknown receptor(s) of white adipocytes and other cells, including myocytes and

\section{Department of Endocrinology and} Metabolism, Shandong University Affiliated Jinan Central Hospital, Linong Road 8, 5-I-80I, Jinan 2500I3, People's

Republic of China

Tel +86-15318816220

Email yinxiao@sdu.edu.cn 
hepatocytes, ${ }^{1}$ so potentially mediating the beneficial effects of physical activity. ${ }^{2}$ Irisin has been proposed to affect obesity and metabolism by increasing adipocyte browning and beta-cell insulin secretion, ${ }^{3-5}$ therefore irisin was discussed as a potential treatment option in obesity and type 2 diabetes (T2DM). However, clinical and functional studies on the association of irisin with obesity and glucose metabolic status remain controversial in a population with different ages and races. Most, but not all studies show a positive association between irisin and indices of adiposity, ${ }^{6}$ but there was data concerning the Chinese Han population ${ }^{7}$ and Korean prepubertal children $^{8}$ which showed the opposite results. It seems that diverse confounders may have affected the results of different clinical studies. ${ }^{9}$ Ethnicity emerged as a determinant in the association between adiposity, glucose tolerance status and irisin. ${ }^{1}$ Because irisin is still an appealing molecule from a pathophysiological point of view and a therapeutic target for obesity and metabolic disease, so far data concerning the Chinese population was limited. We recruited a middle-aged Chinese Han race population with diverse adiposity and glucose tolerance status in this study to elucidate the relationship between circulating irisin and obesity, as well as glucose metabolic indices and other cardiovascular risk factors. Physical activity is an important factor to influence irisin level in human, ${ }^{6}$ the physical activity scores were matched in different groups of this study to avoid bias.

\section{Methods}

\section{Subjects}

This cross-sectional single-center study recruited 740 participants with residence in Jinan, a city in northern China. The inclusion criteria were: $30-50$ years old, wide range of body mass index (BMI), no diabetes or newly diagnosed (duration of diabetes less than 2 years) drug naïve T2DM. A complete medical examination was carried out before the inclusion in the study, and individuals were excluded if they had an acute illness, liver or renal disfunction, myocardial infarction or thyroid disease. They should not be using any medication (such as anti-diabetic, hypotensive drugs, lipid-lowering drugs, exogenous insulin, antiinflammatory, thyroid hormone) that would interfere with the results. Participants could not be involved in regular exercise programs during the previous 12 months according to the Baecke Habitual Physical Activity Questionnaire. $^{10}$
This study was conducted in accordance with the Declaration of Helsinki. The institutional Ethics Committee (Jinan Central Hospital) approved the study protocol, and each participant signed an approved informed consent form. Enrolment into the study occurred between October and December in 2019 in Jinan Central Hospital. Participants were divided into four subgroups according to body mass index (BMI) and glucose tolerance condition: normal-weight group (NW group; $\mathrm{BMI}<25 \mathrm{~kg}$ / $\mathrm{m}^{2}$ without T2DM), overweight/obese group (OB group; BMI $\geq 25 \mathrm{~kg} / \mathrm{m}^{2}$, without T2DM), normal-weight diabetes group (DM-NW group; BMI $<25 \mathrm{~kg} / \mathrm{m}^{2}$ with T2DM), overweight/obese diabetes group (DM-OB group; BMI $\geq$ $25 \mathrm{~kg} / \mathrm{m}^{2}$, with T2DM). T2DM were defined according to the most recent consensus statements. ${ }^{11}$

Physical activity was assessed from questionnaire concerning the type and frequency of exercise before the enrollment, including: work category and work time per week, if they did structured physical exercise, what kind of physical exercise, duration of the exercise and exercise time per week. Trained investigators obtained metabolic equivalent of task (MET) of specific work and activity based on the Compendium of Physical Activities, ${ }^{12}$ and found the physical activity score (PA score $=$ MET $\times$ duration of the activity) of participants.

\section{Anthropometric, Body Composition Measurements}

Height, body weight and waist circumference were measured, the BMI was calculated as body weight $(\mathrm{kg}) /$ height $^{2}$ $\left(\mathrm{m}^{2}\right)$. Systolic and diastolic arterial blood pressure were measured by physicians according to standardized procedures. Body composition, including fat free mass (FFM), fat mass (FM) measurements were performed by bioelectrical impedance analysis (BIA) (TANITA MC-180, Japan).

\section{Laboratory Analysis}

Participants underwent blood sampling for the assessment of blood chemistry and hormonal parameters. The quantitative measurement of irisin in human serum samples was performed using a commercial enzyme linked immunosorbent assay (ELISA) kit (Irisin ELISA Kit EK-067-52; Phoenix Pharmaceuticals Inc., CA) according to the manufacturer's instructions. Basal insulin was analyzed by an immunoradiometric assay (BioSource International, Camarillo, CA, USA) in a Beckman Coulter (Fullerton, 
CA, USA). Enzymatic colorimetric assay was used to measure fasting plasma glucose (FPG) (Hexokinase activity assay, Abcam), total cholesterol (Cholesterol Gen.2, Roche), high-density lipoproteins (HDL) cholesterol (LDL-Cholesterol plus 2nd generation, Roche), triglycerides (Triglycerides/Glycerol Blanked, Roche) concentrations using a Dimension Autoanalyzer (Cobas501, Roche, Switzerland). The homeostatic model assessment index was calculated following the formulas: HOMA-IR = fasting serum insulin (FINS, $\mathrm{mU} / \mathrm{L}) \times$ fasting plasma glucose $(\mathrm{FPG}, \quad \mathrm{mmol} / \mathrm{L}) / 22.5 ; \quad \mathrm{HOMA}-\mathrm{IS}=1 /(\mathrm{FPG}$ $(\mathrm{mmol} / \mathrm{L}) \times$ FINS $(\mathrm{mIU} / \mathrm{L}))$. Low-density lipoprotein (LDL) cholesterol serum concentration was calculated with Friedewald's formula.

\section{Statistical Analysis}

The normal distribution of the variables was tested using the Kolmogorov-Smirnov tests. The irisin levels exhibited abnormal distribution. ANOVA was used for normal distribution continuous variables, Kruskal-Wallis Test was used for abnormal distribution continuous variables, and $\chi 2$ test was used for categorical variables to compare the difference in groups. The potential association between anthropometric and metabolic parameters with irisin levels was evaluated using the Spearman coefficient test. Multivariate linear regression models (stepwise) were fitted to explain the variations in the circulating irisin levels. Data were reported as the mean $\pm \mathrm{SD}$, and confidence intervals $(95 \% \mathrm{CI})$ were used to describe the linear coefficient (B) values. The statistical analysis was performed using SPSS version 20.0 software (SPSS Inc., Chicago, IL) for Windows XP (Microsoft, Redmond, WA). $P \leq 0.05$ was considered as statistically significant.

\section{Results}

A total of 740 participants (374 males and 366 females) were recruited in this study ( $\mathrm{NW}$ group, $\mathrm{n}=194$; OB group, $\mathrm{n}=196 ;$ DM-NW group, $\mathrm{n}=168$; DM-OB group, $\mathrm{n}=182$ ). Sex, age and PA scores were matched for the four groups. The average value of irisin serum concentrations in the cohort was $8.80 \pm 5.85 \mathrm{ng} / \mathrm{mL}$ (range: 1.24-18.32; median value: 5.21; IQ range: 1.78), and the distribution was abnormal as reported in Figure 1. Women had higher serum irisin concentrations than men $(\mathrm{P}=0.007)$. The DM group has higher HbAlc, FPG and TG level compared to NW and OB groups ( $\mathrm{P}<0.01)$, OB and DM-OB groups have a higher BMI and body fat level compared to NW and DM-NW groups $(\mathrm{P}<0.01)$. The physical, biochemical and clinical characteristics of participants divided according to obesity and glucose tolerance were reported in Table 1.

Obese participants tended to exhibit a higher mean irisin concentration compared to normal weight individuals, in either non-diabetes (OB group 18.28 $\pm 10.9 \mathrm{ng} /$ $\mathrm{mL}$ vs $\mathrm{NW}$ group $7.91 \pm 4.34 \mathrm{ng} / \mathrm{mL}, \mathrm{p}<0.01)$ or the T2DM population (DM-OB group 10.21 $\pm 4.49 \mathrm{ng} / \mathrm{mL}$ vs DM-NW $5.66 \pm 2.92 \mathrm{ng} / \mathrm{mL}, \mathrm{p}<0.01)$. Irisin levels were lower in diabetes patients compared to a person without diabetes, in either normal weight (DM-NW group 5.66 $\pm 2.92 \mathrm{ng} / \mathrm{mL}$ vs NW group $7.91 \pm 4.34 \mathrm{ng} / \mathrm{mL}, \mathrm{P}<0.01$ ) or obese population (DM-OB group $10.21 \pm 4.49 \mathrm{ng} / \mathrm{mL}$ vs OB group 18.28 $\pm 10.9 \mathrm{ng} / \mathrm{mL}, \mathrm{P}<0.01)$.

Using Spearman correlations coefficient to compare all the participants in this study cohort, there was a significant positive correlation between serum irisin concentrations and BMI $(\mathrm{r}=0.627, \mathrm{p}<0.01)$, fat mass $(\mathrm{kg})(\mathrm{r}=0.446$, $\mathrm{p}<0.01)$, fat Mass $(\%)(\mathrm{r}=0.22, \mathrm{p}<0.01)$, waist circumference $(\mathrm{r}=0.27, \mathrm{p}<0.01)$, DBP $(\mathrm{r}=0.09, \mathrm{p}=$ $0.012)$, fasting plasma insulin $(r=0.73, p<0.01)$, HOMAIR index $(r=0.607, p<0.01)$, serum concentrations of LDL-C $(\mathrm{r}=0.153, \mathrm{p}<0.01)$, triglyceride $(\mathrm{r}=0.211, \mathrm{p}<$ $0.01)$ and total cholesterol $(\mathrm{r}=0.135, \mathrm{p}<0.01)$, and a significant inverse correlation between serum irisin concentrations and $\mathrm{HbA1c}(\mathrm{r}=-0.175, \mathrm{p}<0.01)$, fasting plasma glucose $(\mathrm{r}=-0.174, \mathrm{p}<0.01)$, and HOMA-IS index $(\mathrm{r}=-0.608, \mathrm{p}<0.01)$ in this study population, no significant correlation was found between blood concentrations of irisin and SBP and serum concentrations of LDL-C (Table 2), there was also no significant correlation between irisin concentration and age, which may be due to the narrow age range (30-50 years old) in this middleaged population.

To further investigate the potential factors involved in the changes in the circulating irisin levels, a multivariate linear regression (stepwise) with significant factors of Table 2 was done, and the analysis showed that BMI, HbA1c, HOMA-IR, HOMA-IS and sex had an independent influence on the circulating irisin concentration (Table 3). Weight, fat mass (\%), fasting plasma glucose and insulin were not included in the model because of the collinearity. The results indicated that the circulating irisin level increased $1 \mathrm{ug} / \mathrm{mL}$ as BMI increased $0.8 \mathrm{~kg}$ / $\mathrm{m}^{2}$, HbAlc decreased $1.17 \%$ and HOMA IS decreased 1.55. Females had a higher circulating irisin level compared to the male sex $(\mathrm{B}=1.29, \mathrm{P}=0.003)$. The corrected $\mathrm{R}^{2}$ of the model was 0.465 . These results 

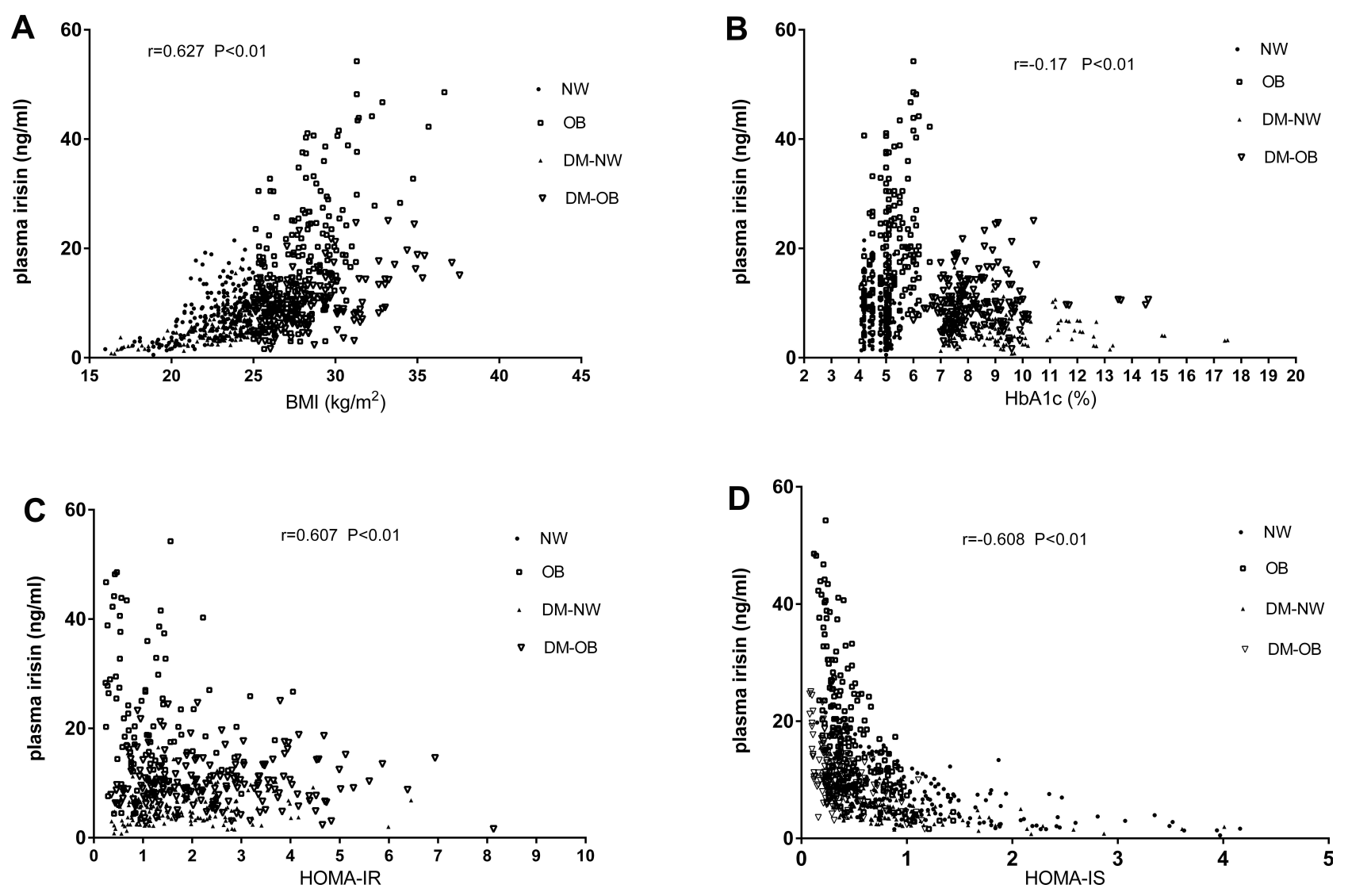

Figure I The correlation of plasma irisin with BMI (A), HbAIc (B), HOMA-IR (C) and HOMA-IS (D).

Abbreviations: BMI, body mass index; DM-NW, diabetes with normal weight group; DM-OB, diabetes with overweight/obese group; HOMA-IR, homeostasis model assessment for insulin resistance index; HOMA-IS, homeostasis model assessment for insulin sensitivity index; NW, normal weight group; OB, overweight and obese group.

indicated that both adiposity, glucose level and insulin resistance status influence the irisin circulating levels in the Chinese middle-aged population.

\section{Discussion}

The current study, performed in middle-aged Chinese individuals with a wide range of BMI and glucose tolerance status, confirmed the positive correlation of circulating irisin with parameters of adiposity including body weight, BMI, waist circumference and fat mass, and insulin resistance index in this physical activity matched population, this work also contributed to valuable information by showing that circulating irisin levels correlated negatively with $\mathrm{HbA1c}$ and insulin sensitivity index. These results indicated that adipose tissue may be a primary inducer of irisin secretion in addition to muscle tissue, especially in obesity, the results also indicated the physiological function of irisin to improve glucose tolerance, which was often impaired in obesity, but the circulating irisin levels declined in patients with overt diabetes.
Several human studies focused on the potential correlations between circulating irisin levels and obesity. Most, but not all, studies have reported that circulating irisin is positively associated with BMI and weight. ${ }^{1,13-20}$ This association remains positive also in extremely obese phenotypes. Moreover, irisin is positively associated with fat mass, waist circumference, waist-to-hip ratio and muscle mass, ${ }^{13,15,17,21}$ similar results were also found in the Asian population. ${ }^{20,22}$ Irisin levels paralleled body weight reduction after weight-loss by either dietary or surgical treatment, and restored after regaining the lost weight, ${ }^{13}$ which revealed that irisin strongly reflects adiposity and body fat mass. But there are also the opposite conclusions concerning the relation between the circulating irisin and obesity, studies of Korean prepubertal children ${ }^{8}$ and Brazil obese middle-aged men $^{23}$ observed inverse correlations between irisin and body mass index, a study in Chinese adults also indicated that serum irisin were correlated negatively with BMI, and was a predictor of insulin resistance. ${ }^{7}$ The results about correlation between circulating irisin and obesity was still controversial, especially in 
Table I The Demographic and Clinical Characteristics of the Subjects

\begin{tabular}{|c|c|c|c|c|}
\hline Group & NW & OB & DM-NW & DM-OB \\
\hline $\mathrm{n}$ & 194 & 196 & 168 & 182 \\
\hline $\operatorname{Sex}(M / F)$ & $99 / 95$ & $97 / 99$ & $86 / 82$ & $92 / 90$ \\
\hline Age (years) & $36.80 \pm 5.86$ & $37.8 I \pm 5.58$ & $38.30 \pm 5.05$ & $37.48 \pm 4.75$ \\
\hline Height $(\mathrm{cm})$ & $168.16 \pm 7.39$ & $169.01 \pm 7.46$ & $165.96 \pm 6.06^{*}$ & $165.44 \pm 5.87$ \\
\hline Weight (kg) & $64.06 \pm 7.93$ & $79.61 \pm 9.78^{*}$ & $62.19 \pm 7.1$ & $77.90 \pm 9.36 * \#$ \\
\hline$W C(\mathrm{~cm})$ & $84.4 \pm 4.67$ & $100.2 \pm 8.76^{* \#}$ & $88.34 \pm 6.68$ & $98.34 \pm 9.27^{* \#}$ \\
\hline BMI $\left(\mathrm{kg} / \mathrm{m}^{2}\right)$ & $22.60 \pm 1.86$ & $27.80 \pm 2.15^{* \#}$ & $22.55 \pm 1.97$ & $28.41 \pm 2.59 * \#$ \\
\hline Fat mass (kg) & $16.42 \pm 4.20$ & $24.69 \pm 6.53^{* \#}$ & $18.50 \pm 5.06 *$ & $27.66 \pm 7.17^{* \#}$ \\
\hline Fat mass (\%) & $25.44 \pm 4.80$ & $30.94 \pm 6.6 I^{*}$ & $29.46 \pm 6.29 *$ & $35.26 \pm 6.70 * \#$ \\
\hline PA score & $24.99 \pm 2.24$ & $25.04 \pm 2.24$ & $25.11 \pm 2.26$ & $24.97 \pm 2.20$ \\
\hline $\mathrm{SBP}(\mathrm{mmHg})$ & $121.84 \pm 17.65$ & $128.42 \pm 17.25^{* \#}$ & $135.55 \pm 20.58^{*}$ & $|35.8| \pm 19.02 *$ \\
\hline $\mathrm{DBP}(\mathrm{mmHg})$ & $74.92 \pm 11.27$ & $79.66 \pm 12.19 *$ & $79.24 \pm 11.62 *$ & $77.95 \pm 10.60 *$ \\
\hline HbAlc (\%) & $4.77 \pm 0.44$ & $5.15 \pm 0.58 *$ & $9.07 \pm 1.87^{*}$ & $8.36 \pm 1.34 *$ \\
\hline Fins $(\mathrm{mU} / \mathrm{L})$ & $5.78 \pm 3.42$ & $11.27 \pm 5.28^{* \#}$ & $4.48 \pm 2.94 *$ & $8.84 \pm 5.02^{* \#}$ \\
\hline FPG $(\mathrm{mmol} / \mathrm{L})$ & $5.20 \pm 0.71$ & $5.22 \pm 0.6 \mathrm{I}^{* \#}$ & $9.36 \pm 2.16^{*}$ & $9.42 \pm 1.90$ *\# \\
\hline HOMA-IR & $1.35 \pm 0.90$ & $2.62 \pm 1.28^{* \#}$ & $1.80 \pm 1.1 I^{*}$ & $3.72 \pm 2.30$ *\# \\
\hline HOMA-IS & I.II \pm 0.77 & $0.49 \pm 0.26$ *\# & $0.82 \pm 0.59 *$ & $0.37 \pm 0.23^{* \#}$ \\
\hline LDL-C (mmol/L) & $2.92 \pm 0.74$ & $3.03 \pm 0.9 I^{\#}$ & $2.53 \pm 0.78^{*}$ & $2.99 \pm 1.14^{\#}$ \\
\hline HDL-C (mmol/L) & $1.44 \pm 0.4 \mathrm{I}$ & $1.21 \pm 0.33 *$ & I.14 $140.46 *$ & $1.18 \pm 0.4 I^{*}$ \\
\hline Triglyceride $(\mathrm{mmol} / \mathrm{L})$ & $1.24 \pm 0.66$ & $1.89 \pm 1.21^{* \#}$ & $1.53 \pm 0.84 *$ & $2.07 \pm 1.28^{* \#}$ \\
\hline Total cholesterol $(\mathrm{mmol} / \mathrm{L})$ & $4.62 \pm 0.82$ & $4.64 \pm 1.02^{\#}$ & $4.19 \pm 1.00 *$ & $4.90 \pm 1.29^{\#}$ \\
\hline Irisin (ng/mL) & $7.91 \pm 4.34$ & $18.28 \pm 10.9 * \#$ & $5.66 \pm 2.92 *$ & $10.21 \pm 4.49 * \#$ \\
\hline
\end{tabular}

Notes: Data showed as mean \pm SD. ANOVA was used for normal distribution continuous variables, Kruskal-Wallis test was used for abnormal distribution continuous variables, and $\chi 2$ test was used for categorical variables to compare the difference among four groups. ${ }^{*} \mathrm{P}<0.0 \mathrm{I}$ compared to $\mathrm{NW}$ group; ${ }^{*} \mathrm{P}<0.0 \mathrm{I}$ compared to $\mathrm{DM}-\mathrm{NW}$ group.

Abbreviations: NW, normal weight group; OB, overweight and obese group; DM-NW, diabetes with normal weight group; DM-OB, diabetes with overweight/obese group; WC, waist circumference; BMI, body mass index; WC, waist circumference; SBP, systolic blood pressure; DBP, dialectic blood pressure; Fins, fasting plasma insulin; FPG, fasting plasma glucose; HOMA-IR, homeostatic model assessment of insulin resistance; HOMA-IR, homeostatic model assessment of insulin sensitivity; LDL-C, lowdensity lipoprotein cholesterol; HDL-C, high-density lipoproteins cholesterol.

the eastern-Asian population. The results of this study revealed that overweight and obese individuals had higher circulating irisin levels in either diabetes and non-diabetes groups compared with a lean person, circulating irisin was positively correlated with adiposity parameters in the middle-aged Chinese population. This study indicated that the irisin circulating levels were conditioned by adiposity level, so in addition to muscle mass, fat mass was also the main contributor to the variability of the irisin circulating levels in this middle-aged Chinese population.

We also examined the association between circulating irisin level and insulin sensitivity in this study cohort. Previous studies found a weak but significant positive association of circulating irisin with insulin resistance in nondiabetic subjects, ${ }^{24}$ fasting blood glucose has been generally positively associated with irisin levels, ${ }^{14}$ but acute changes in glucose or insulin levels, as during an OGTT, do not affect irisin levels. Ethnic variability might affect the association, since insulin sensitivity indices show an inverse association with irisin in non-diabetic people of Asiatic and American origin, ${ }^{24,25}$ but a positive association was reported in a European study. ${ }^{26}$ The present results showed the negative association between irisin level and insulin sensitivity indices in both non-diabetes subgroup and the whole study cohort, which was also supported by its positive association with insulin resistance as assessed by the insulin resistance index, these results were in accordance with the previous study in a non-diabetes population. Contrary to a previous study which showed that circulating irisin levels were negatively associated with insulin resistance in diabetes, ${ }^{27}$ we did not find such negative association between serum irisin level and insulin resistance index in our diabetes subgroup population, because serum irisin levels were negatively correlated with diabetes duration, ${ }^{28}$ the result might be due to the newly-diagnosed middle aged diabetes patients we recruited in the diabetes subgroup population. Previous animal studies ${ }^{5}$ showed irisin has potentially multiple favorable effects on glucose homeostasis and insulin sensitivity by promoting energy expenditure and glucose uptake, ${ }^{29}$ the increase of irisin under conditions 
Table 2 Spearman Correlations Among Irisin, Metabolic and Anthropometric Variables

\begin{tabular}{|c|c|c|}
\hline & \multicolumn{2}{|l|}{ Irisin } \\
\hline & Rho & $P$ \\
\hline Sex & 0.105 & 0.004 \\
\hline Age (years) & -0.002 & 0.95 \\
\hline BMI $\left(\mathrm{kg} / \mathrm{m}^{2}\right)$ & 0.627 & $<0.01$ \\
\hline$W C(\mathrm{~cm})$ & 0.27 & $<0.01$ \\
\hline Fat mass $(\mathrm{kg})$ & 0.446 & $<0.01$ \\
\hline Fat mass $(\%)$ & 0.22 & $<0.01$ \\
\hline $\mathrm{SBP}(\mathrm{mmHg})$ & 0.03 & 0.32 \\
\hline $\mathrm{DBP}(\mathrm{mmHg})$ & 0.09 & 0.012 \\
\hline $\mathrm{HbAIc}(\%)$ & -0.17 & $<0.01$ \\
\hline Fins (mU/L) & 0.73 & $<0.01$ \\
\hline FPG (mmol/L) & -0.17 & $<0.01$ \\
\hline HOMA-IR & 0.607 & $<0.01$ \\
\hline HOMA-IS & -0.608 & $<0.01$ \\
\hline LDL-C (mmol/L) & 0.153 & $<0.01$ \\
\hline $\mathrm{HDL}-\mathrm{C}(\mathrm{mmol} / \mathrm{L})$ & -0.06 & 0.84 \\
\hline Triglyceride $(\mathrm{mmol} / \mathrm{L})$ & 0.211 & $<0.01$ \\
\hline Total cholesterol (mmol/L) & 0.135 & $<0.01$ \\
\hline
\end{tabular}

Abbreviations: Rho, Spearman correlation coefficients; BMI, body mass index; WC, waist circumference; Fins, fasting plasma insulin; FPG, fasting plasma glucose; HOMA-IR, homeostatic model assessment of insulin resistance; HOMA-IR, homeostatic model assessment of insulin sensitivity; LDL-C, low-density lipoprotein cholesterol; HDL-C, high-density lipoproteins cholesterol.

Table 3 Independent Effects of Body Composition and Metabolic Factors on Variations in Irisin Levels

\begin{tabular}{|l|l|l|l|}
\hline & B & 95\% Confidence Interval & P \\
\hline BMI $\left(\mathrm{kg} / \mathrm{m}^{2}\right)$ & 0.80 & $0.62-0.97$ & $<0.00 \mathrm{I}$ \\
HbAIc $(\%)$ & -1.17 & $(-1.37)-(-0.97)$ & $<0.00 \mathrm{I}$ \\
HOMA-IR & 1.29 & $0.94-1.64$ & $<0.00 \mathrm{I}$ \\
HOMA-IS & -1.55 & $(-2.58)-(-0.52)$ & 0.003 \\
Sex & 1.29 & $0.42-2.14$ & 0.003 \\
\hline \multicolumn{2}{|l|}{ Corrected $\mathrm{R}^{2}=0.465$} & & 0.003 \\
\hline
\end{tabular}

Note: Using multivariable linear regression analysis with stepwise method. Abbreviations: BMI, body mass index; HOMA-IR, homeostatic model assessment of insulin resistance; HOMA-IR, homeostatic model assessment of insulin sensitivity.

of obesity may indicate a physiological function to improve glucose tolerance which is often impaired in obese subjects. A recent study in obese Chinese children also showed that irisin level changes may indicate the early stage of cardiovascular disease, ${ }^{20}$ which is also partly due to the close relationship between insulin resistance and cardiovascular disease.

There was a wide range of glucose tolerance status in this study cohort, the association between T2DM and irisin was also detected. Although a few studies suggested that higher irisin levels are related to a better glucose metabolic profile and lower risk of T2DM development in obese/ overweight population, ${ }^{14,19}$ the majority of the studies concerning a T2DM population have reported lower irisin levels in the population with T2DM than controls, ${ }^{30,31}$ and this association was more pronounced in Asian than European populations, since the contradictory findings found slightly higher irisin levels in Caucasian ${ }^{32}$ and Saudi Arabian ${ }^{33}$ T2DM patients compared to the control, which indicated ethnicity emerged as a determinant in the relationship between T2DM and irisin. In accordance with the previous studies, our data showed that circulating irisin levels were obviously lower in the middle-aged Chinese population with T2DM compared to adiposity matched controls, and were negatively correlated with $\mathrm{HbAlc}$ devices in the current study cohort. We found that circulating irisin levels in obese diabetes patients were lower versus obese non-diabetes people, and higher compared to normal weight T2DM patients, which suggests that the incremental secretion of irisin is compensatory in order to provide a counterbalance to metabolic disturbances in obese populations, but it seems likely that the compensatory secretion of irisin progressed to a secretion failure once diabetes developed. Our results also showed the serum irisin levels in obese T2DM was weak but significantly higher than lean healthy individuals, for serum irisin levels were negatively correlated with diabetes duration as mentioned above, ${ }^{28}$ the results might indicate that, compared to the glucose status, muscle and fat mass were still the main contributor for circulating irisin level in this newly-diagnosed middle aged diabetes population.

The advantage of this study was that we recruited individuals with a wide range of adiposity and glucose tolerance status to investigate the correlation of circulting irisin with obesity, glucose level, insulin sensitivity and other cardiovascular risk factors in a physical activity matched middle-aged Chinese population. There was a limitation that data regarding bone health and PTH levels was not included in this study, as the existence of an interplay between PTH and irisin metabolism has been suggested in previous reports. ${ }^{34,35}$ There is also an issue needing cautious interpretation, the quality of commercially available ELISA kits for circulating irisin was skepticized, it is better to validate the irisin values toward tandem mass spectrometry, which was considered to be the gold standard method. ${ }^{36}$

In conclusion, this study confirmed that circulating irisin was associated with adiposity, glucose tolerance and insulin 
resistance status in a middle-aged Chinese population, the increase of irisin under conditions of obesity may indicate its physiological function to improve glucose tolerance which is often impaired in obese subjects, but this compensatory secretion of irisin seems likely to be progressing to a secretion failure once diabetes develops. Irisin may have potentially favorable effects on glucose homeostasis and insulin sensitivity, but the specific receptor and potential signal pathway through which irisin could protect against obesity needs to be further explored.

\section{Statement of Ethics}

Subjects have given their written informed consent. The study protocol has been approved by the local Ethical and Research Committee of Jinan Central Hospital on human research.

\section{Acknowledgments}

We thank LetPub (www.letpub.com) for its linguistic assistance during the preparation of this manuscript.

\section{Author Contributions}

All authors made a significant contribution to the work reported, whether that is in the conception, study design, execution, acquisition of data, analysis and interpretation, or in all these areas; took part in drafting, revising or critically reviewing the article; gave final approval of the version to be published; have agreed on the journal to which the article has been submitted; and agree to be accountable for all aspects of the work.

\section{Funding}

The study was supported by funding from the Natural Science Foundation of China (81300686) and the Key Research and Development Program of Shandong Province (2018GSF118184). The sponsors had no influence on the design, conduct, interpretation or publication of this work.

\section{Disclosure}

The authors have no conflicts of interest to declare.

\section{References}

1. Perakakis N, Triantafyllou GA, Fernandez-Real JM, et al. Physiology and role of irisin in glucose homeostasis. Nat Rev Endocrinol. 2017;13 (6):324-337.

2. Yuksel Ozgor B, Demiral I, Zeybek U, et al. Effects of irisin compared with exercise on specific metabolic and obesity parameters in female mice with obesity. Metab Syndr Relat Disord. 2020;18(3):141-145. doi: $10.1089 /$ met.2019.0083
3. Lee $\mathrm{P}$, Linderman JD, Smith $\mathrm{S}$, et al. Irisin and FGF21 are cold-induced endocrine activators of brown fat function in humans. Cell Metab. 2014;19(2):302-309. doi:10.1016/j.cmet.2013.12.017

4. Natalicchio A, Marrano N, Biondi G, et al. The myokine irisin is released in response to saturated fatty acids and promotes pancreatic beta-cell survival and insulin secretion. Diabetes. 2017;66 (11):2849-2856. doi:10.2337/db17-0002

5. Polyzos SA, Anastasilakis AD, Efstathiadou ZA, et al. Irisin in metabolic diseases. Endocrine. 2018;59(2):260-274.

6. Buscemi S, Corleo D, Vasto S, et al. Factors associated with circulating concentrations of irisin in the general population cohort of the ABCD study. Int $J$ Obes. 2018;42(3):398-404. doi:10.1038/ ijo.2017.255

7. Liu BW, Yin FZ, Qi XM, Fan DM, Zhang Y. The levels of serum irisin as a predictor of insulin resistance in han chinese adults with metabolically healthy obesity. Clin Lab. 2017;63(5):881-886. doi:10.7754/Clin.Lab.2016.160805

8. Shim YS, Kang MJ, Yang S, Hwang IT. Irisin is a biomarker for metabolic syndrome in prepubertal children. Endocr J. 2018;65 (1):23-31. doi:10.1507/endocrj.EJ17-0260

9. Cooke AB, Gomez YH, Daskalopoulou SS. 5 years later: irisin detection still an issue. Eur $J$ Endocrinol. 2017;177(6):C1-C4. doi:10.1530/EJE-17-0572

10. Bauman A, Ainsworth BE, Sallis JF, et al. The descriptive epidemiology of sitting. A 20-country comparison using the International Physical Activity Questionnaire (IPAQ). Am J Prev Med. 2011;41 (2):228-235. doi:10.1016/j.amepre.2011.05.003

11. Garber AJ, Abrahamson MJ, Barzilay JI, et al. Consensus statement by the American Association of Clinical Endocrinologists and American College of Endocrinology on the Comprehensive Type 2 Diabetes Management Algorithm - 2018 Executive Summary. Endocr Pract. 2018;24(1):91-120. doi:10.4158/CS-2017-0153

12. Ainsworth BE, Haskell WL, Herrmann SD, et al. 2011 Compendium of physical activities: a second update of codes and MET values. Med Sci Sports Exerc. 2011;43(8):1575-1581. doi:10.1249/MSS.0b013 e31821ece12

13. Crujeiras AB, Pardo M, Arturo RR, et al. Longitudinal variation of circulating irisin after an energy restriction-induced weight loss and following weight regain in obese men and women. Am J Hum Biol. 2014;26(2):198-207. doi:10.1002/ajhb.22493

14. Huerta AE, Prieto-Hontoria PL, Fernandez-Galilea M, et al. Circulating irisin and glucose metabolism in overweight/obese women: effects of alpha-lipoic acid and eicosapentaenoic acid. J Physiol Biochem. 2015;71(3):547-558. doi:10.1007/s13105-015$0400-5$

15. Loffler D, Muller U, Scheuermann K, et al. Serum irisin levels are regulated by acute strenuous exercise. J Clin Endocrinol Metab. 2015;100(4):1289-1299. doi:10.1210/jc.2014-2932

16. Pardo M, Crujeiras AB, Amil M, et al. Association of irisin with fat mass, resting energy expenditure, and daily activity in conditions of extreme body mass index. Int $J$ Endocrinol. 2014;2014:857270. doi: $10.1155 / 2014 / 857270$

17. Stengel A, Hofmann T, Goebel-Stengel M, Elbelt U, Kobelt P, Klapp BF. Circulating levels of irisin in patients with anorexia nervosa and different stages of obesity-correlation with body mass index. Peptides. 2013;39:125-130. doi:10.1016/j.peptides.2012.1 1.014

18. Palacios-Gonzalez B, Vadillo-Ortega F, Polo-Oteyza E, et al. Irisin levels before and after physical activity among school-age children with different BMI: a direct relation with leptin. Obesity. 2015;23 (4):729-732. doi:10.1002/oby.21029

19. Sahin-Efe A, Upadhyay J, Ko BJ, et al. Irisin and leptin concentrations in relation to obesity, and developing type 2 diabetes: a cross sectional and a prospective case-control study nested in the Normative Aging Study. Metabolism. 2018;79:24-32. 
20. Yin $\mathrm{C}, \mathrm{Hu} \mathrm{W}$, Wang $\mathrm{M}, \mathrm{Lv} \mathrm{W}$, Jia $\mathrm{T}$, Xiao Y. Irisin as a mediator between obesity and vascular inflammation in Chinese children and adolescents. Nutr Metab Cardiovasc Dis. 2020;30(2):320-329. doi:10.1016/j.numecd.2019.09.025

21. Crujeiras AB, Zulet MA, Lopez-Legarrea $P$, et al. Association between circulating irisin levels and the promotion of insulin resistance during the weight maintenance period after a dietary weight-lowering program in obese patients. Metabolism. 2014;63 (4):520-531. doi:10.1016/j.metabol.2013.12.007

22. Jang HB, Kim HJ, Kang JH, Park SI, Park KH, Lee HJ. Association of circulating irisin levels with metabolic and metabolite profiles of Korean adolescents. Metabolism. 2017;73:100-108. doi:10.1016/j. metabol.2017.05.007

23. Bonfante ILP, Chacon-Mikahil MPT, Brunelli DT, et al. Obese with higher FNDC5/Irisin levels have a better metabolic profile, lower lipopolysaccharide levels and type 2 diabetes risk. Arch Endocrinol Metab. 2017;61(6):524-533. doi:10.1590/2359-3997000000305

24. Qiu S, Cai X, Yin H, et al. Association between circulating irisin and insulin resistance in non-diabetic adults: a meta-analysis. Metabolism. 2016;65(6):825-834. doi:10.1016/j.metabol.20 16.02 .006

25. Moreno-Perez O, Reyes-Garcia R, Munoz-Torres M, et al. High irisin levels in nondiabetic HIV-infected males are associated with insulin resistance, nonalcoholic fatty liver disease, and subclinical atherosclerosis. Clin Endocrinol (Oxf). 2018;89(4):414-423. doi:10.1111/cen.13800

26. Moreno-Navarrete JM, Ortega F, Serrano M, et al. Irisin is expressed and produced by human muscle and adipose tissue in association with obesity and insulin resistance. J Clin Endocrinol Metab. 2013;98(4): E769-E778. doi:10.1210/jc.2012-2749

27. Maciorkowska M, Musialowska D, Malyszko J. Adropin and irisin in arterial hypertension, diabetes mellitus and chronic kidney disease. Adv Clin Exp Med. 2019;28(11):1571-1575. doi:10.17219/acem/ 104551
28. Khorasani ZM, Bagheri RK, Yaghoubi MA, et al. The association between serum irisin levels and cardiovascular disease in diabetic patients. Diabetes Metab Syndr. 2019;13(1):786-790. doi:10.1016/j. dsx.2018.11.050

29. Zhang Y, Li R, Meng Y, et al. Irisin stimulates browning of white adipocytes through mitogen-activated protein kinase p38 MAP kinase and ERK MAP kinase signaling. Diabetes. 2014;63(2):514-525. doi:10.2337/db13-1106

30. Duran ID, Gulcelik NE, Unal M, et al. Irisin levels in the progression of diabetes in sedentary women. Clin Biochem. 2015;48 (18):1268-1272. doi:10.1016/j.clinbiochem.2015.07.098

31. Li Z, Wang G, Zhu YJ, et al. The relationship between circulating irisin levels and tissues AGE accumulation in type 2 diabetes patients. Biosci Rep. 2017;37(3). doi:10.1042/BSR20170213

32. Garcia-Fontana B, Reyes-Garcia R, Morales-Santana S, et al. Relationship between myostatin and irisin in type 2 diabetes mellitus: a compensatory mechanism to an unfavourable metabolic state? Endocrine. 2016;52(1):54-62. doi:10.1007/s12020-015-0758-8

33. Al-Daghri NM, Mohammed AK, Al-Attas OS, et al. SNPs in FNDC5 (irisin) are associated with obesity and modulation of glucose and lipid metabolism in Saudi subjects. Lipids Health Dis. 2016;15:54. doi:10.1186/s12944-016-0224-5

34. Palermo A, Sanesi L, Colaianni G, et al. Between irisin and PTH: from basic studies to clinical evidence in hyperparathyroidism. J Clin Endocrinol Metab. 2019;104(8):3088-3096. doi:10.1210/jc.201802216

35. Palermo A, Strollo R, Maddaloni E, et al. Irisin is associated with osteoporotic fractures independently of bone mineral density, body composition or daily physical activity. Clin Endocrinol (Oxf). 2015;82(4):615-619. doi:10.1111/cen.12672

36. Jedrychowski MP, Wrann CD, Paulo JA, et al. Detection and quantitation of circulating human irisin by tandem mass spectrometry. Cell Metab. 2015;22(4):734-740. doi:10.1016/j.cmet.2015.08.001

\section{Publish your work in this journal}

Diabetes, Metabolic Syndrome and Obesity: Targets and Therapy is an international, peer-reviewed open-access journal committed to the rapid publication of the latest laboratory and clinical findings in the fields of diabetes, metabolic syndrome and obesity research. Original research, review, case reports, hypothesis formation, expert opinion and commentaries are all considered for publication. The manuscript management system is completely online and includes a very quick and fair peer-review system, which is all easy to use. Visit http://www.dovepress.com/testimonials.php to read real quotes from published authors.

Submit your manuscript here: https://www.dovepress.com/diabetes-metabolic-syndrome-and-obesity-targets-and-therapy-journal 